\title{
Synthesis and characterisation of spiropyran-polymer brushes in micro-capillaries: towards an integrated optical sensor for continuous flow analysis
}

\author{
Larisa Florea, Alexandre Hennart, Dermot Diamond, Fernando Benito-Lopez ${ }^{*}$ \\ CLARITY: Centre for Sensor Web Technologies, National Centre for Sensor Research, School of Chemical Sciences, \\ Dublin City University, Dublin 9, Ireland.
}

\begin{abstract}
Fused silica-capillaries were successfully functionalised with spiropyran-polymer brushes using spiropyran functionalised norbornyl derivative as monomer. The polymerisation was achieved by surface-initiated ringopening metathesis polymerisation. A three-dimensional arrangement, covalently attached to the inner wall of the fused-silica capillary, was obtained. The spiropyran moiety has the freedom to open and close in response to light (ultraviolet, white light) within the polymer brushes. The coating was fully characterised by Scanning Electron Microscopy, absorbance measurements and kinetic studies. The photo-response of the coatings showed very good reproducibility comparable with spiropyran monomers in solution demonstrating that this platform can be used for the develop of capillary integrated sensors based on the inherited sensing proprieties of spiropyran moieties.
\end{abstract}

Keywords: spiropyran, micro-capillary, ROMP, sensor, continous flow

\section{Introduction}

Previously reported capillary optical sensors have shown numerous advantages compared to planar optical sensors as such a) are suitable for direct sampling, b) cheap fabrication protocols (c) represent an optical waveguide structure that enables various methods of optical interrogation, d) have small internal volumes and e) very rapid response times.[1, 2]

The development of capillary optical sensors involves the coating of the inner walls of a capillary (usually glass) with a sensitive layer that can be optically interrogated. Welgl et al.[1] reported capillary optical sensors obtained by immobilising a carbon dioxide sensitive layer onto the inner walls of a capillary by "reverse pumping" meanwhile, other research groups have published integrated-optic ammonia sensors based as well in capillaries as the sensor platform.[3, 4] Lippitsch et al. [5] showed the use of capillary optical sensors for biomedical applications proving good sensor performance and easy handling by the integration of the sample compartment, the optical sensor, and the light-collecting optics into a single device. However, most of the reported protocols involved non-covalent bonding of the sensitive material to the inner walls of the capillary[3,5] and required protective coatings[1] to avoid leaching of the immobilised sensitive material over time[6].

An attractive alternative for making capillary integrated optical sensors is the possibility of incorporating active sensing surfaces that can be switched on and off remotely using light. In our laboratories, we are extensively working in the generation of switchable optical sensors based on photo-responsive materials using spiropyran derivatives[7-10].

Spiropyrans are one of the most popular classes of photocromic compounds as they are particularly interesting targets for the development of new approaches for sensing. They offer new routes to multifunctional materials that take advantage of their photo-reversible interconversion between two thermodynamically stable states: a spiropyran (SP) form, and a merocyanine (MC) form, Figure 1, which have dramatically different charge, polarity and molecular conformations[11]. Spiropyrans are an attractive starting point in the construction of molecular-level sensors with molecular recognition properties and signal transduction ability due to their unique molecule binding power and signal transduction function[12]. The MC may interact with the surrounding environment (solvent or analyte) manifesting different photochromic responses[12]. Based on these sensing proprieties, that are only 
shown by the MC form, spiropyrans have been employed in analytical chemistry as molecular sensors where the sensing behaviour can be remotely switched ON/OFF using light of appropriate wavelengths (by switching between the spiro (OFF) and the merocyanine (ON) form). The sensing capability of spiropyrans in solution have been extensively studied and demonstrated for a wide range of analytes from metal ions[13-17], neutral molecules (such as nucleobases[18], amino acids[19, 20] and DNA[21]), a range of anions[22, 23] and solvents of different polarities[24].

The possibility of fabricating optical sensors that can be photo-activated has significant implications in science since this methodology represents a totally non-invasive manner of controlling and interrogating the response of the sensor. In this respect, a significant amount of effort has been devoted to functionalise surfaces[9] and micro-fluidic platforms[25] with photochromic materials. Rosario et al.[26] coated capillary tubes with a photoresponsive monolayer based on spiropyran and showed that the water level inside the capillary rised when the light source was switched from VIS to UV in correlation with the switching of the surface-bound spiropyran molecule between nonpolar and polar forms. Previous work done in our group by Benito-Lopez et al.[25] showed that micro-fluidic channels coated with spiropyran monolayers can be used as photonically controlled self-indicating systems for metal ion accumulation and release, based on the metal ion-binding and molecular recognition properties of the MC form. However, the main disadvantage of the monolayer approach is the low concentration of photochromic moieties, which manifests in extremely weak absorbance peaks, where the formation of the MC form cannot be detected by eye[26].

In the context of developing chemical sensors, the use of homopolymers, where each monomer contains the spiropyran unit, is desired as it increases the concentration of the photochromic component in the polymer[27] and enhances the photochromic processes[28]. For instance, Samanta et al.[28] showed that when glass slides are coated with spiropyran polymer brushes obtained by surface-initiated ring opening metathesis polymerisation (SI-ROMP), the photochromic behaviour is greatly enhanced compared to the monolayer. Moreover, the color and wettability of the surface can be externally controlled using light as the external stimulus.

In this context, we are focusing our research in the synthesis and characterisation of sensing coatings based on spiropyran moieties inside micro-capillaries that can be externally photo-controlled in a non-invasive way. Functionalisation of the inner wall of a fused silica capillary with this photoresponsive molecule provides a convenient small platform for rapid analysis and detection. Furthermore, continuous flow operation facilitates real-time measurements and consequently fast analysis protocols[25].

We have successfully coated fused-silica micro-capillaries with photochromic polymer brushes based on spiropyran moieties using surface-initiated ring-opening metathesis polymerisation (SiROMP). Their synthesis offers a transition from a two-dimensional to a three-dimensional arrangement and allows for high density of functional groups to be obtained in a limited area[28]. The photoresponsive behaviour of the spiropyran polymer brushes was fully characterised using acetonitrile $(\mathrm{ACN})$ as the running solvent in the capillary.
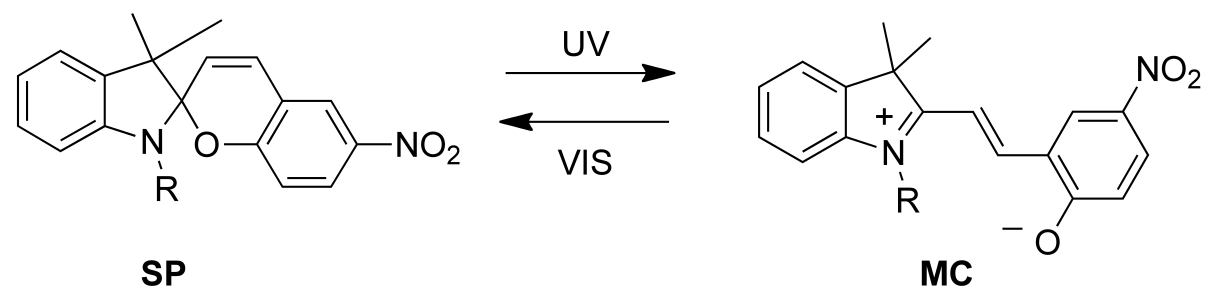

Fig.1. Molecular structure of a generic spiropyran moiety irradiated with UV/white light, spiropyran (left) and merocyanine (right).

\section{Experimental}

\subsection{Materials}

7-Octenyltrichlorosilane (Gelest), 5-norbornene-2-carboxylic acid, mixture of isomers (Alfa Aesar), 1-(2-Hydroxyethyl)-3,3-dimethylindolino-6'-nitrobenzopyrylospiran (SP1) (TCI Europe), $N, N^{\prime}$ - 
Dicyclohexylcarbodiimide (DCC) (Aldrich), 4-(Dimethylamino)pyridine (DMAP) (Aldrich) and Grubbs Generation-II catalyst (Aldrich) were used as received. Acetone, acetonitrile, dry tetrahydrofuran (THF), dry toluene and dry dichloromethane $\left(\mathrm{CH}_{2} \mathrm{Cl}_{2}\right)$ solvents were purchased from Aldrich and used as received. Fused-silica capillaries (100 $\mu \mathrm{m}$ ID, $375 \mu \mathrm{m}$ OD) were purchased from Polymicro Technologies (Phoenix, AZ, USA).

\subsection{Procedures}

\subsubsection{Synthesis of spiropyran functionalised norbornene monomer}

Norbornyl functionalised spiropyran monomer[28] (SP-M) was prepared, as described in Fig. 2, from the reaction of 5-norbornene-2-carboxylic acid (mixture of isomers) with SP1 in the presence of DCC and DMAP in THF, according to the previously reported method[27]. A representative example of this method is the following: SP1 $(0.3 \mathrm{~g}, 0.851 \mathrm{mmol})$ and 5-norbornene-2-carboxylic acid $(0.104$ $\mathrm{ml}, 0.851 \mathrm{mmol}$ ) were placed in a $250 \mathrm{~mL}$ round-bottomed flask. After the solution was dissolved in dry THF $(20 \mathrm{~mL})$ and cooled to $0{ }^{\circ} \mathrm{C}$, a solution of DCC $(0.21 \mathrm{~g}, 1.02 \mathrm{mmol})$ and DMAP $(0.010 \mathrm{~g}, 0.08$ mmol) in THF ( $5 \mathrm{~mL})$ was added drop-wise over the course of $1 \mathrm{~h}$. The mixture was stirred at $0{ }^{\circ} \mathrm{C}$ for an additional $2 \mathrm{~h}$ and then gradually warmed to $25{ }^{\circ} \mathrm{C}$ over the course of $24 \mathrm{~h}$. During the warming period, a dicyclohexyl urea precipitate formed, which was filtered and washed with THF (3 x $50 \mathrm{~mL})$. After the filtrate was evaporated, the resulting red wax was purified using silica gel column chromatography in the dark with a mixture of hexane/ethylacetate $(10 / 1)$.

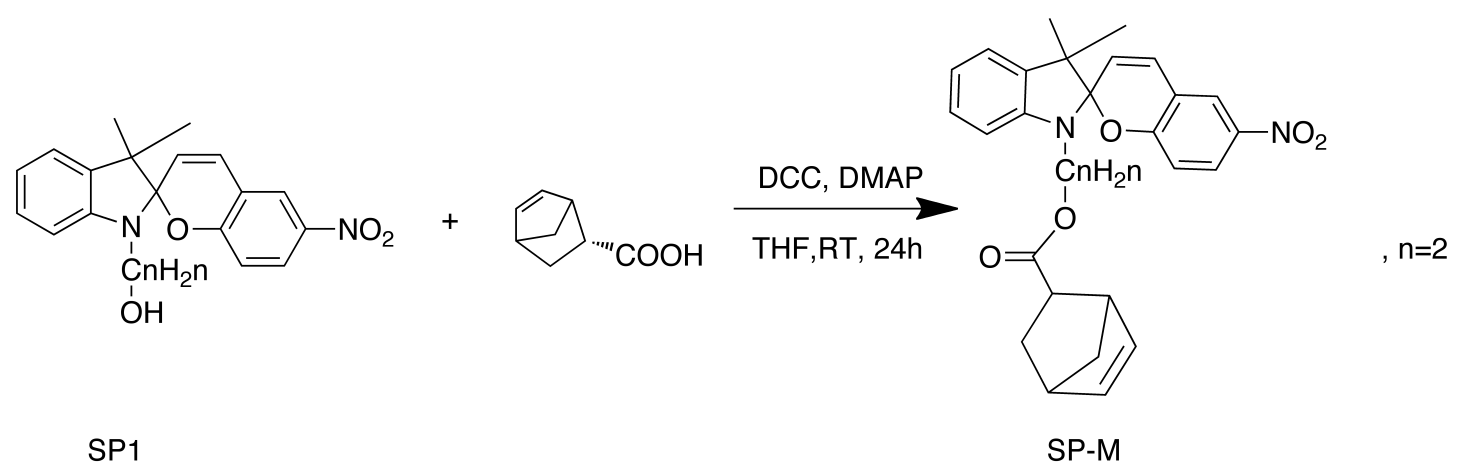

Fig. 2. Synthesis of the spiropyran functionalised norbornene monomer.

\subsubsection{Characterisation of spiropyran functionalised norbornene monomer}

Fourier transform-infrared (FT-IR) measurements were taken with a Perkin Elmer Spectrum GX FT-IR System ${ }^{\circledR}$ instrument equipped with an attenuated total reflectance accessory at 264 scans with 4 $\mathrm{cm}^{-1}$ resolution. A solution of spiropyran norbornene monomer $\left(10^{-2} \mathrm{M}\right)$ in acetone was prepared, irradiated for 1 minute with white light and $1 \mathrm{ml}$ of this solution was transfer onto the ATR crystal. The solvent was then evaporated and the spectrum of the spiropyran norbornene monomer in its closed form was recorded. Next, the ATR crystal was exposed to UV light for 20 seconds to allow complete conversion of the spiropyran norbornene monomer to the open merocyanine form and a new spectrum was recorded.

\subsubsection{Fused silica micro-capillaries coating protocol.}

The protocol used to coat micro-capillaries with spiropyran polymer brushes is described in Fig. 3, steps 1-4. Prior to functionalisation, the inner surface of fused-silica capillaries was first activated with 7-octenyl trichlorosilane. The inner capillary surface was quickly washed with acetone and water, then flushed with a solution of $\mathrm{NaOH} 0.2 \mathrm{M}$ for $30 \mathrm{~min}$ at a flow rate of $0.25 \mu \mathrm{l} / \mathrm{min}$ using a syringe pump, 
and then rinsed with deionised water. Next, the capillary was flushed with a solution of $\mathrm{HCl} 0.2 \mathrm{M}$ for $30 \mathrm{~min}$ at a flow rate of $0.25 \mu \mathrm{l} / \mathrm{min}$, rinsed with water, and with dry toluene. A $0.1 \mathrm{M}$ solution of the silanisation agent (7-octenyl trichlorosilane) $0.1 \mathrm{M}$ in dry toluene was pumped through the capillaries for $90 \mathrm{~min}$ at a flow rate of $0.25 \mu \mathrm{l} / \mathrm{min}$ (Fig. 3 - step 1). The capillaries were then washed with acetone, dried under nitrogen stream, and left at room temperature for $24 \mathrm{~h}$. Later, the capillary was filled with a solution of Grubbs Catalyst Second Generation $0.02 \mathrm{M}$ in degassed $\mathrm{CH}_{2} \mathrm{Cl}_{2}$, closed at both ends using rubber septa and put in a water bath for $1 \mathrm{~h}$ at $45{ }^{\circ} \mathrm{C}$ (Fig. 3 - step 2). After the catalystattached capillary was thoroughly washed with degassed $\mathrm{CH}_{2} \mathrm{Cl}_{2}$. Finally, the capillary filled with a solution of spiropyran functionalised monomer, SP-M $0.5 \mathrm{M}$ in degassed $\mathrm{CH}_{2} \mathrm{Cl}_{2}$, closed at both ends using rubber septa and put in a water bath for at $50{ }^{\circ} \mathrm{C}$ for $4 \mathrm{~h}$ (Fig. 3 - step 3). The polymerisation was quenched by passing ethyl vinyl ether into the capillary (Fig. 3 - step 4). Finally the capillary was thoroughly washed with acetone to remove any physisorbed materials.
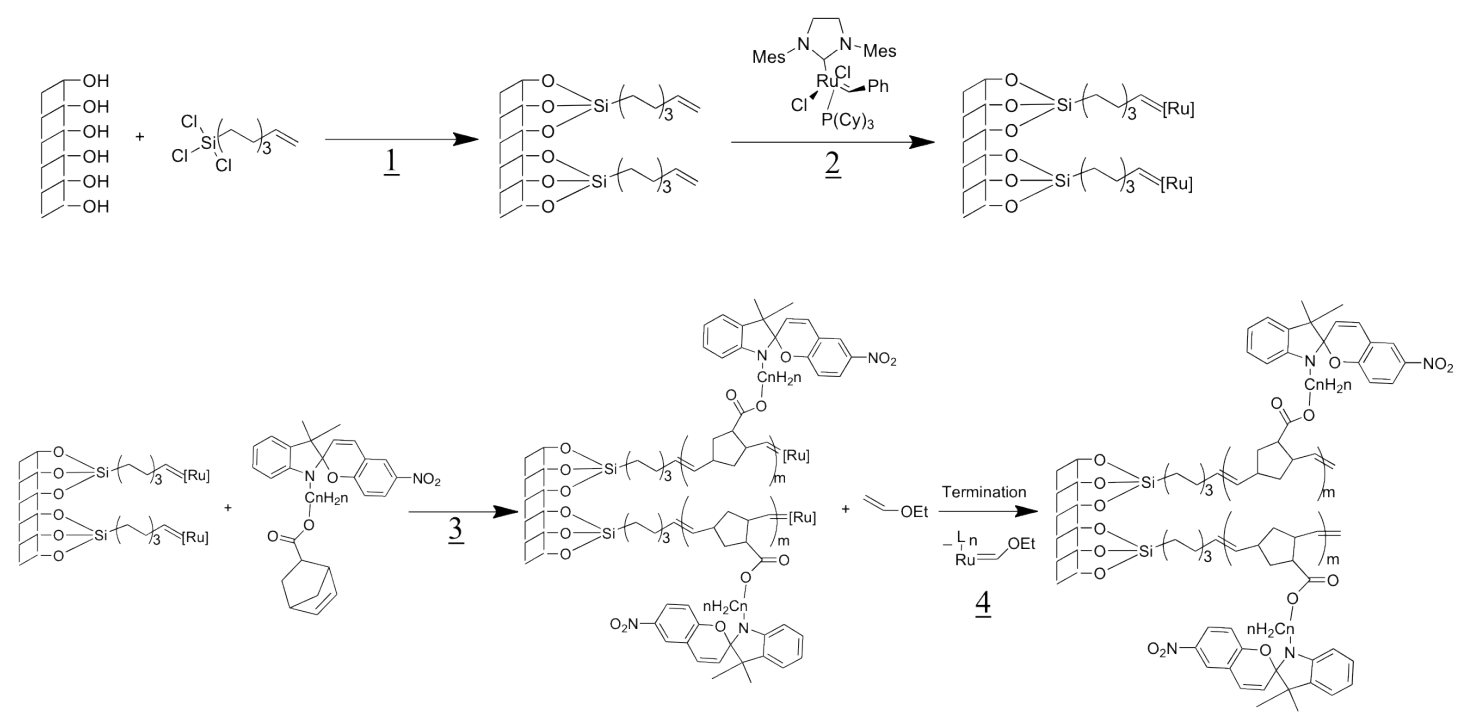

Fig. 3. Functionalisation protocol of fused silica micro-capillaries with spiropyran based polymeric brushes. 


\subsubsection{Estimation of the molar extinction coefficient of the SP-M}

To estimate the molar extinction coefficient $(\varepsilon)$ of the SP-M in acetonitrile (ACN), 5 solutions of different concentration of SP-M $\left(0.2 \times 10^{-5}, 0.4 \times 10^{-5}, 0.6 \times 10^{-5}, 0.8 \times 10^{-5}\right.$ and $\left.10^{-4}\right)$ were prepared. The solutions were irradiated with UV light for 90 seconds using an array of 9 UV LEDs (365 nm) and the spectra were recorded on a UV-Vis-NIR PerkinElmer Lambda 900 spectrometer. A plot of the Absorbance at $\lambda_{\max }(\mathrm{nm})$ versus concentration showed good linearity $\left(\mathrm{R}^{2}=0.990\right)$. The molar extinction coefficient of SP-M was calculated based on the Lambert-Beer law (eq.1), where the path length, $l$, was $1 \mathrm{~cm}$ (the width of the quartz cuvette).

\subsubsection{Physical morphology characterisation of polymer brushes}

Spiropyran polymer brushes were imaged using scanning electron microscopy (SEM) performed on a Carl Zeiss EVOLS 15 system at an accelerating voltage of $4.27 \mathrm{kV}$. The micro-capillaries were cut using a SGT capillary column cutter with rotating diamond blade (SHORTIX, Nederland) to create a smooth cut of the capillary wall. Then they were placed in vertical position in a custom made metallic capillary holder that has holes of internal diameters equal to the external diameter of the microcapillary $(375 \mu \mathrm{m})$. This set-up allows the micro-capillaries to be kept in vertical position. During the imaging process, the stage was tilted of an angle between $0-15^{\circ}$ for better imaging of the inner wall of the micro-capillary.

\subsubsection{Optical characterisation of spiropyran coatings}

The UV irradiation source used to switch the spiropyran based coatings and the spiropyran monomer (SP-M) solution was an array of 9 UV LEDs placed at a distance of $1 \mathrm{~cm}$ from the spiropyran solution or the functionalised micro-capillaries, respectively. The LEDs have an emission wavelength peak at $365 \mathrm{~nm}$ and an optical output power of $1.2 \mathrm{~mW}$, supplied by Roithner Lasertechnik Austria. The white light irradiation source used for the switching of MC back to SP form (in both solutions and capillary coatings) was a DC-regulated light source supplied by Polytec, USA, and placed at a distance of $1 \mathrm{~cm}$ from the illuminated area. The maximum power output of the lamp is $150 \mathrm{~W}$, and the intensity control of the light output was fixed at $50 \%$. The optical switching of the spiropyran moiety inside the capillary has been studied using USB 4000 Fiber Optic Spectrometer - Ocean Optics, Inc, when the light source was a LS-1 tungsten halogen lamp (white light) obtained from Ocean Optics, Inc. A representation of the set-up used for absorbance measurements is described in Fig. 4. The solvent acetonitrile (ACN) was passed through the capillary at constant flow rate $(0.5 \mu \mathrm{l} / \mathrm{min})$ using a syringe pump (PHD 2000 Syringe) purchased from Harvard Apparatus. All the absorbance spectra were smoothed using Origin Software (each initial spectra contained 3900 points; the smoothing was realised automatically using an average value for every 50 points).

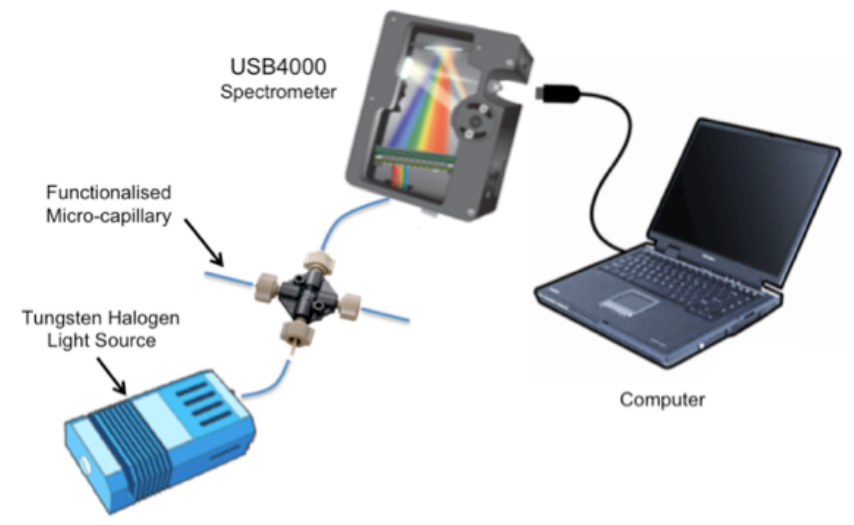

Fig. 4. Scheme of the set-up used for absorbance measurements. 


\section{Results and discussions}

\subsection{Structure characterization and photoinduced conversion of SP to MC}

ATR-FT-IR was used to characterise the chemical structure of the spiropyran functionalised monomer as well as the photo-induced conversion of spiropyran to merocyanine in solution. Figure 5 shows the ring-closed SP and ring-opened MC form of SP-M after irradiation with UV light. Complete characterisation and assignments are listed in Table 1. Bands of particular importance are the C-C-N bend that appears in the spectra of SP-M at $1026 \mathrm{~cm}^{-1}$ and the O-C-N stretching emerging at $952 \mathrm{~cm}^{-1}$. These bands disappear upon irradiation with UV light and new bands appear at 1593, 1426, and 1307 $\mathrm{cm}^{-1}$ assigned to the $\mathrm{C}=\mathrm{N}^{+}, \mathrm{C}-\mathrm{O}^{-}$and $\mathrm{C}-\mathrm{N}^{+}$stretches, respectively, characteristic to the open merocyanine form[29-35]. Also the symmetrical stretching band of the nitro group is shifted to lower energy from 1520 to $1509 \mathrm{~cm}^{-1}$ upon irradiation, due to the increased conjugation brought about in the planar, merocyanine[35].
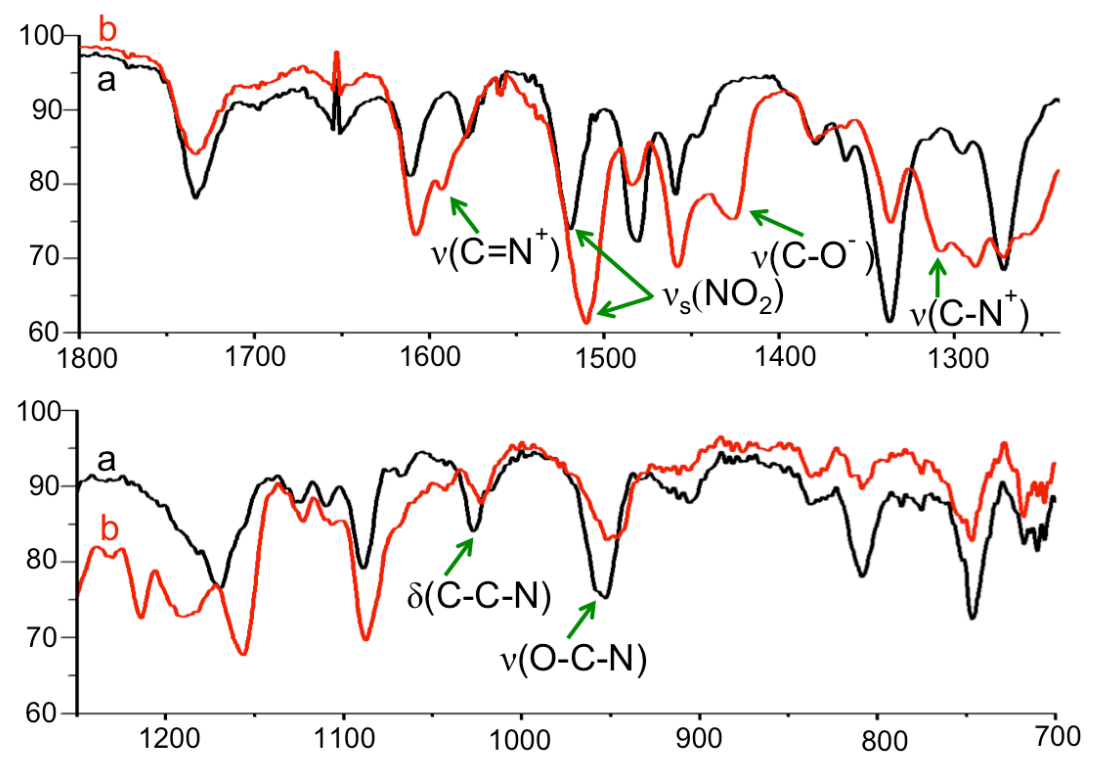

Fig. 5. FT-IR spectra of spiropyran functionalised norbornene monomer (SP-M) before UV irradiation (a) and after UV irradiation (b). Peaks that indicate the photoconversion of spiropyran to merocyanine are labeled. 
Table 1. Important FT-IR frequencies for spiropyran functionalised norbornene monomer (SP-M) before (spiropyran) and after (merocyanine) UV irradiation.

\begin{tabular}{|l|l|l|}
\hline & Spiropyran & Merocyanine \\
\hline Assignment & $\begin{array}{l}\text { Wavenumber } \\
\left(\mathrm{cm}^{-1}\right)\end{array}$ & $\begin{array}{l}\text { Wavenumber } \\
\left(\mathrm{cm}^{-1}\right)\end{array}$ \\
\hline $\mathrm{C}=\mathrm{O}$ & 1734 & 1734 \\
\hline $\mathrm{C}=\mathrm{C}$ stretch & 1610 & 1607 \\
\hline $\mathrm{C}=\mathrm{N}^{+}$ & - & 1593 \\
\hline $\mathrm{NO}_{2}$ sym stretch & 1520 & 1509 \\
\hline $\mathrm{C}-\mathrm{C}$ aromatic ring stretch & 1481,1459 & 1483,1457 \\
\hline $\mathrm{C}-\mathrm{O}^{-}$ & & 1426 \\
\hline NO ${ }_{2}$ asym stretch & 1336 & 1336 \\
\hline $\mathrm{C}-\mathrm{N}$ stretch & 1336 & - \\
\hline $\mathrm{C}-\mathrm{N}^{+}$ & - & 1307 \\
\hline $\mathrm{C}-\mathrm{O}-\mathrm{C}$ ether sym stretch & 1271 & - \\
\hline $\mathrm{C}-\mathrm{O}-\mathrm{C}$ ether asym stretch & 1169 & - \\
\hline $\mathrm{C}-\mathrm{O}$ ester stretch & 1169 & 1156 \\
\hline $\mathrm{C}-\mathrm{O}$ ester stretch & 1086 & 1087 \\
\hline $\mathrm{C}-\mathrm{C}-\mathrm{N}$ bend & 1026 & - \\
\hline O-C-N stretch & 952 & - \\
\hline $\begin{array}{l}\mathrm{C}=\mathrm{CH} ; \mathrm{CH} \text { out of plane } \\
\text { deformation (cis) }\end{array}$ & 952 & 952 \\
\hline C-H bending & 809 & 809 \\
\hline $\mathrm{C}-\mathrm{H}$ bending & 746 & 746 \\
\hline $\mathrm{C}-\mathrm{H}$ bending & 709 & 709 \\
\hline & & \\
\hline
\end{tabular}

\subsection{Polymer brushes length analysis}

Fused silica capillaries were covalently functionalised with spiropyran polymer brushes as described in the experimental section. Scanning electron microscopy of the inner wall of the capillary showed a 3D arrangement of polymer brushes with a thickness of about 1-2 $\mu \mathrm{m}$ as shown in Figure 6 . Samanta et al.[28] also used surface-initiated ring-opening metathesis polymerisation to produce spiropyran polymeric brushes on a flat glass surface, obtaining brushes length of approximately $120 \mathrm{~nm}$ which are shorter than the ones obtained by us. This length enhanced effect during the synthesis of polymer brushes inside capillaries was previously reported by Miller et al. [36] who showed that when atom transfer radical polymerisation (ATRP) is employed to produce polymer brushes inside a capillary, the length of the brushes is longer than when the same technique was employed on flat surfaces. Although the coatings present good homogeneity over the cross section, certain heterogeneity was observed along the length of the micro-capillary through colourless areas on the capillary walls when irradiation with UV light (coloured MC form should be present). Further investigations are on going to explore ways to synthesise more homogenous coatings.

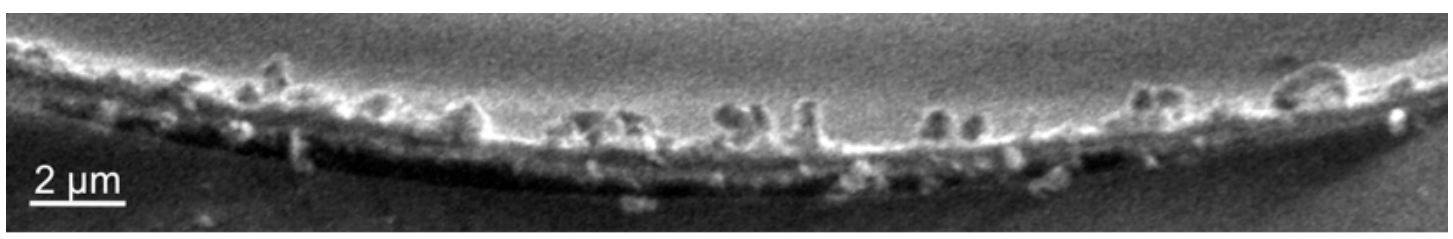

Fig. 6. Scanning electron microscopy image of the inner wall of a fused silica capillary (100 $\mu \mathrm{m}$ inner diameter $)$ after functionalisation with spiropyran polymer brushes. 


\subsection{Photo-patterning of spiropyran-merocyanine forms in the capillary}

SP-M and SP-polymer brushes were optically characterised in acetonitrile (ACN) since it is one of the most common solvents to study the photophysical properties of spiropyran and spiropyran derivatives before and after UV irradiation[37]. In our experimental conditions, ACN was used because it is able to dissolve the monomer and solvate the polymer brushes, while also inhibiting the formation of aggregates.

ACN was passed through the functionalised capillary at a constant flow rate of $5 \mu \mathrm{Lmin}^{-1}$ filling the channel completely and avoiding the generation of air bubbles. Then masks formed from metal foil were placed in different positions along the capillary in order to pattern open/closed spiropyran regions along the capillary (see Figure $7 \mathrm{a}-\mathrm{b})$. To do this, the capillary was irradiated with UV light $(365 \mathrm{~nm})$ for $10 \mathrm{~s}$ through the mask. After removing the mask it is clearly visible, even by eye, that the parts of the capillary exposed to UV light irradiation have a strong purple colour characteristic of the merocyanine form while the portions that were covered by the mask remained colourless depicting the presence of the colourless closed form of the spiropyran, Fig. 7c. Moreover, when the entire capillary is exposed to white light irradiation, the colour pattern of the capillary recovers the initial colourless spiropyran form, Fig.7d. This protocol demonstrates that, with the polymer brushes structures, the spiropyran presents no steric hindrance to the kinetics of opening and closing in response to UV and white light irradiation, respectively.

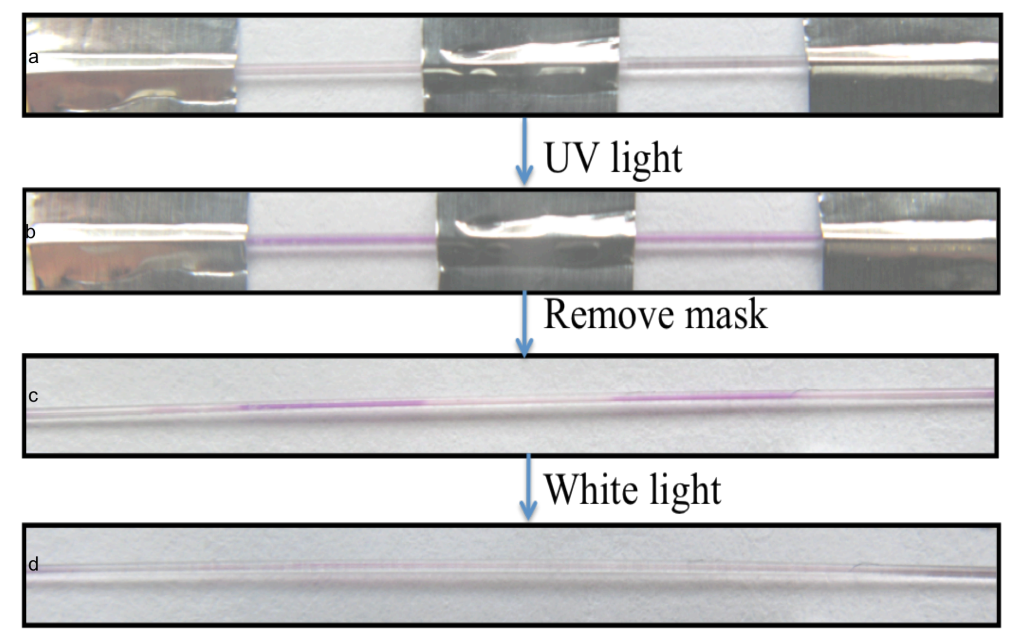

Fig. 7. Pictures of the spiropyran polymeric brushes functionalised capillaries when filled with acetonitrile (ACN) after irradiation for 10 seconds with UV light through a mask ( $a$ and b), c shows the pattern generated by the mask on the capillary. Picture d shows the capillary after irradiation for 1 minute with white light.

\subsection{Characterisation of SP-monomer in solution and SP-polymer brushes in the capillary by UV-Vis spectrometry}

UV-Vis spectra of the polymer brushes were taken using the set-up described in the experimental section, Figure 4, permitting in-situ spectroscopic characterisation of the coatings inside the capillary. When the capillary is irradiated with UV light for $10 \mathrm{~s}$ in the presence of ACN, the absorbance spectra of the coating presents a $\lambda_{\max }$ at about $546 \mathrm{~nm}$. This absorption band disappears after irradiation of the capillary with white light for 1 minute because of the switching of the merocyanine molecule back to the closed spiropyran form (Fig 8a). This behaviour shows a good agreement with the photochromic response of the monomer $\left(10^{-4} \mathrm{M}\right)$ in ACN solution under identical conditions (Fig. 8b), which presents a $\lambda_{\max }$ of $568 \mathrm{~nm}$.

The shift of the $\lambda_{\max }$ in the absorption spectra of the polymer brushes compared to that of the monomer solutions is most likely due to local environmental effects related to the immobilisation of the spiropyran moiety and the integration within the polymer matrix. This effect has been previously observed in different spiropyran-polymer systems, where the shift in the $\lambda_{\max }$ depends on the nature of the polymer and the technique of immobilisation.[37-39] The significant hypsochromic (blue) shift in $\lambda_{\max }$ for the polymer brushes compared to the monomer indicates a more polar micro-environment of the spiropyran in the brushes and it can be explained taking into account the compact organisation of 
spiropyran units in the polymer brushes, where the conformation of a single spiropyran moiety is not only influenced by the solvent but also by the neighbouring spiropyran units that in response to UV light, are present in their more polar merocyanine form.
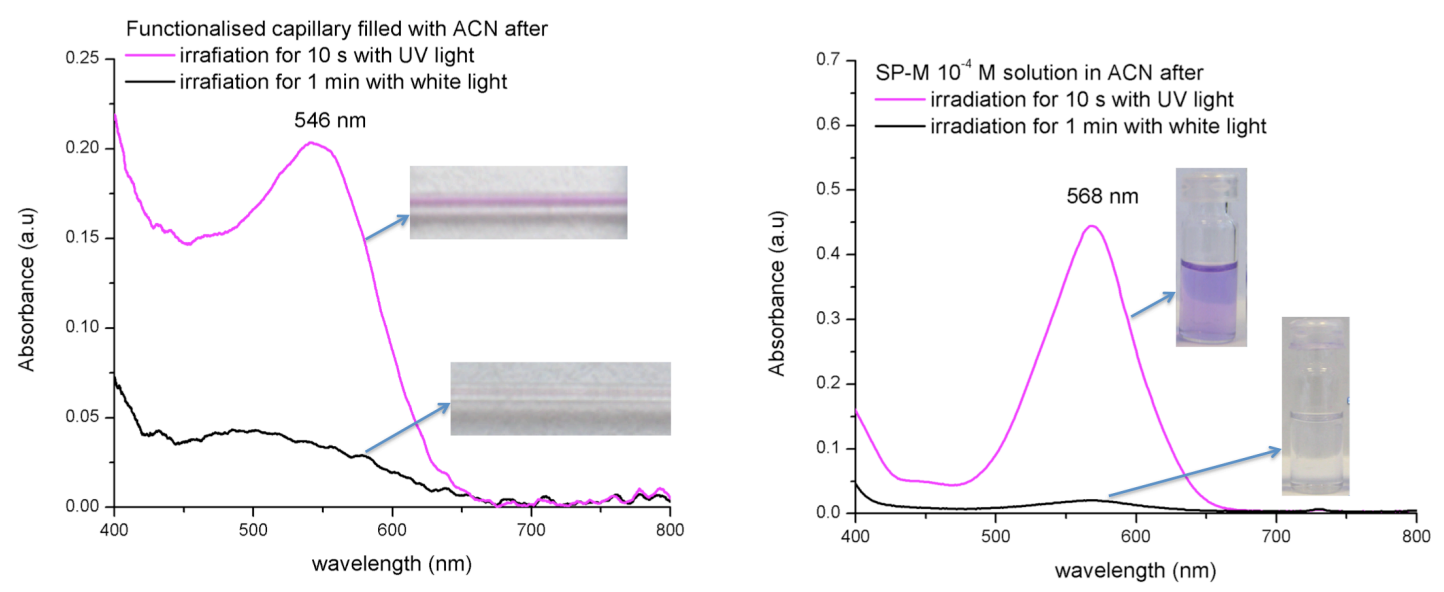

Fig. 8. (a) Absorbance spectra of spiropyran polymer brushes functionalised in the capillary filled with ACN after irradiation with UV and white light, respectively. (b) Absorbance spectra of spiropyran monomer (SP-M) solution $10^{-4} \mathrm{M}$ in ACN after irradiation with UV and white light, respectively. The spectra are accompanied by photos of the functionalised capillary (a) and monomeric solution (b) under the same experimental conditions.

Although the UV-Vis spectra were taken directly in the capillary (100 $\mu \mathrm{m}$, inner diameter) a high absorbance value (0.2 a.u.) of the coating after $10 \mathrm{~s}$ UV light irradiation was obtained, indicating a high loading of spiropyran units on the capillary walls. The concentration of spiropyran units inside the micro-capillary was estimated using the Lambert-Beer law, with the molar extinction coefficient, $\varepsilon$, considered to be equal to the molar extinction coefficient of the monomer in ACN, since the polymer brushes are formed from identical monomeric units. The molar extinction coefficient of the SP-M calculated as described in the experimental section was found to be $46000 \pm 10 \mathrm{M}^{-1} \mathrm{~cm}^{-1}$. Consequently the loading of spiropyran units inside the capillary was estimated to be approximately $43 \times 10^{-3} \mathrm{moldm}^{-3}$, according to Lambert Beer law presented below.

$$
\mathrm{A}_{\text {brushes }}=\varepsilon \cdot l \cdot c
$$

Where $\mathrm{A}_{\text {brushes }}$ is the absorbance of the photochromic coating after exposure for $10 \mathrm{~s}$ to UV light $(\sim 0.2$ a.u. $), \varepsilon$ is the molar extinction coefficient of the monomer in $\operatorname{ACN}\left(46000 \pm 10 \mathrm{M}^{-1} \mathrm{~cm}^{-1}\right), c$ is the concentration of spiropyran units in the capillary and $l$ is the inner diameter of the capillary $(100 \mu \mathrm{m})$.

However, this is the loading of the spiropyran units relative to the capillary and not to the polymeric brushes that represent only 2-4\% of the capillary width. These high values show that this platform can be easily used to produce new types of sensors based on the inherited spiropyran proprieties where the response of the sensor can be optically interrogated by simple absorbance measurements. The strong response of the coating to the photo-stimulus (manifested in high absorbance values) shows the promise of this platform for the development of optofluidics sensors where optic and fluidic functionalities are integrated at the micro-scale to leverage their combined advantages.[40]

\subsection{Switching properties of the SP-polymer brushes in the capillary by UV-Vis spectrometry}

When the colourless SP-polymer brushes are illuminated with UV light $(365 \mathrm{~nm})$ the capillary become purple-coloured, and the colour change is easily observed by eye. This effect has been intensively investigated by us using other polymeric surfaces like poly(methyl methacrylate)[7-9, 41] and in polystyrene/ silica beads[10], onto which spiropyran derivatives were covalently immobilised. The capillary, filled with ACN was irradiated with $365 \mathrm{~nm}$ UV light for 10 seconds to convert the SPpolymer brushes to the MC-polymer brushes form, then, the channels were illuminated with white light 
for 1 minute. Absorbance values at $546 \mathrm{~nm}$ were recorded immediately after irradiation, Fig.9a. The plot clearly shows a high efficient interconversion of spiropyran units inside the polymer brushes. Therefore, the spiropyran moiety has enough freedom and it is well solvated by ACN, allowing the high degree of conformational flexibility required for efficient switching between the MC and SP forms.

Photo-stability over time has to be considered when dealing with spiropyran photochromic dyes, as a well-documented photo-bleaching process[42-44] occurs when SP is exposed to UV-vis radiation for extended time periods. After 5 switching cycles, the efficiency was found to be constant in the capillary. Figure 8a shows typical results obtained for one location on the capillary. The $\mathrm{A}_{\mathrm{SP}}=0.035 \pm$ 0.003 a.u. and the $\mathrm{A}_{\mathrm{MC}}=0.204 \pm 0.003$ a.u. The $\Delta \mathrm{A}_{\mathrm{SP} \rightarrow \mathrm{MC}}=0.169 \pm 0.003$ a.u. and the $\Delta \mathrm{A}_{\mathrm{MC} \rightarrow \mathrm{SP}}=-$ $0.169 \pm 0.001$ a.u. for 5 cycles obtaining similar differences in absorbance values, therefore these cycles are reproducible and repeatable, with no hysteresis. This result demonstrates that the capillary could be re-used at least five times, without significant photo-bleaching, suggesting that it can be photo-switched many more times if required.
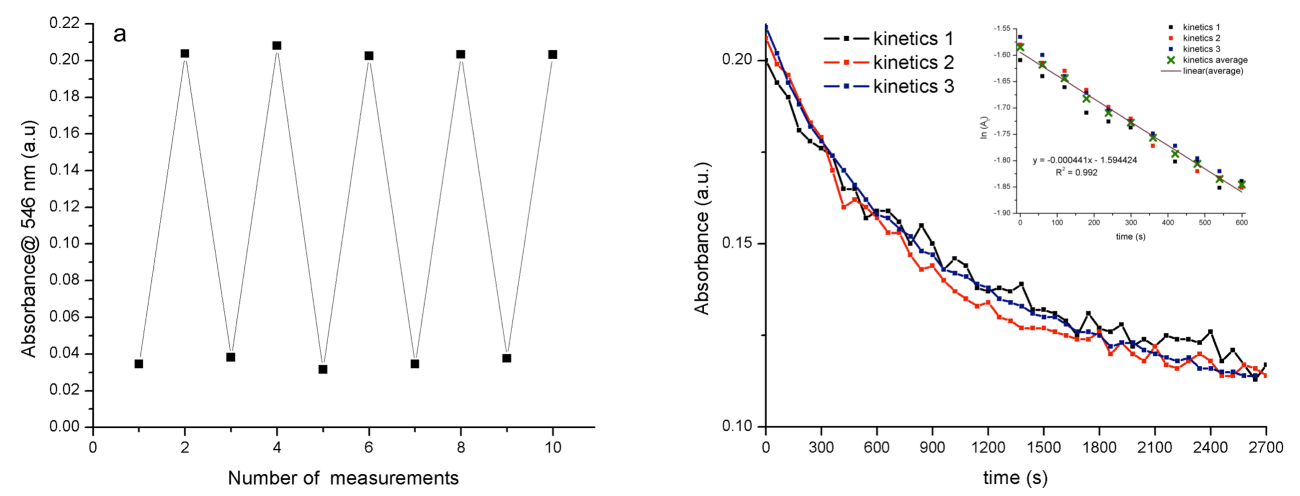

Fig. 9. (a) Absorbance intensity at $546 \mathrm{~nm}$ of spiropyran polymer brushes functionalised in a capillary after repeated switching cycles consisting of 10 seconds UV light exposure followed by 1 minute white light irradiation. (b) Absorbance decreases at the $\lambda_{\max }(546 \mathrm{~nm})$ in acetonitrile as a function of time for the transition of the spiropyran polymeric coating from the merocyanine form, obtained after UV light irradiation, to the closed spiropyran form. Inset - Linear plot of $\ln (\mathrm{At}) v \mathrm{~s}$. time used for the determination of the first order kinetic constant of the spiropyran closing process $(\mathrm{MC} \rightarrow \mathrm{SP})$.

The kinetics of the closing process of the spiropyran moiety inside the polymeric brushes was studied using white light irradiation, Fig.9b. This involved inducing the formation of the MC state by exposure of the capillary to UV light irradiation for 10 seconds, and then monitoring the decrease of the absorbance value at $\lambda_{\max }(546)$ at a fixed time interval of 60 seconds upon removal of the UV light source, as equilibrium is re-established between the SP and MC forms. It is known from solution studies that this process follows first-order kinetics[45], with the thermodynamics and kinetics strongly influenced by the solvent. Figure $9 \mathrm{~b}$ shows the reduction of the absorbance at the $\lambda_{\max }$ for the transition from $\mathrm{MC}$ form of the polymer brushes to the SP form at $18{ }^{\circ} \mathrm{C}$. From these measurements, the firstorder rate constants $k$ for the MC to SP closing process in the presence of white light (coming from LS1 tungsten halogen lamp) was determined by plotting $\ln \left(A_{t}\right)$ versus time and it was found to be $4.4 \pm$ $0.5 \times 10^{-4} \mathrm{~s}^{-1}$. This value is similar to other reported values for spiropyran functionalised polystyrene beads in $\operatorname{ACN}\left(1.5 \times 10^{-4}( \pm 2 \%) \mathrm{s}^{-1}\right)[10]$ or for poly(methyl methacrylate) chains having a single photochromic spiropyran end-group in $\mathrm{ACN}$ at $15^{\circ} \mathrm{C}\left(3.03 \times 10^{-3} \mathrm{~s}^{-1}\right)[37]$.

\section{Conclusions}

To the best of our knowledge this is the first time spiropyran based polymer brushes were grafted on the inner walls of a micro-capillary. Using Si-ROMP a three-dimensional arrangement formed of polymeric brushes, covalently attached to the inner wall of the fused-silica capillary, where the spiropyran moiety has the freedom to open and close in response to light (UV, white light) was obtained. It was shown that the photochromic behaviour presents good reproducibility and the switching between SP to MC can be performed at least 5 times with no photobleaching. The spiropyran 
polymer brushes inherit the spiropyran proprieties, in addition, a good agreement was found between the monomer in solution and photochromic coatings obtained inside the capillary. Based on these promising results, this fabricated and characterised capillary platform based on the spiropyran proprieties will be further investigated as a novel miniaturised integrated optical molecular sensor for real time analysis in continuous flow mode using a variety of analytes.

\section{Acknowledgements}

The project has been carried out with the support of the Irish Research Council for Science, Engineering and Technology (IRCSET) - Embark Initiative and Science Foundation Ireland under grant 07/CE/I1147 and Science Foundation Ireland under the CLARITY award (07/CE/ I1147). We would like to thank Dr. Brendan Twamley for providing the custom designed capillary holder used for SEM imaging.

\section{References}

[1] B.H. Weigl, O.S. Wolfbeis, Capillary optical sensors, Anal. Chem., 66 (1994) 3323-3327.

[2] W.H. Tan, Z.Y. Shi, S. Smith, D. Birnbaum, R. Kopelman, Submicrometer intracellular chemical optical fibres sensors, Science, 258 (1992) 778-781.

[3] J.F. Giuliani, H. Wohltjen, N.L. Jarvis, Reversible optical-waveguide sensor for ammonia vapors, Opt. Lett., 8 (1983) 54-56.

[4] V. Chernyak, R. Reisfeld, R. Gvishi, D. Venezky D., Oxazine-170 in sol-gel glass and PMMA films as a reversible optical waveguide sensor for ammonia and acids, Sensor Mater., 2 (1990) 117126.

[5] M.E. Lippitsch, S. Draxler, D. Kieslinger, H. Lehmann, B.H. Weigl, Capillary waveguide optrodes: An approach to optical sensing in medical diagnostics, Appl. Optics, 35 (1996) 3426-3431.

[6] B. Kuswandi, R. Narayanaswamy, Polymeric encapsulated membrane for optrodes, Fresenius J. Anal. Chem., 364 (1999) 605-607.

[7] R. Byrne, D. Diamond, Chemo/bio-sensor networks, Nat. Mater., 5 (2006) 421-424.

[8] R.J. Byrne, S.E. Stitzel, D. Diamond, Photo-regenerable surface with potential for optical sensing, J. Mater. Chem., 16 (2006) 1332-1337.

[9] A. Radu, S. Scarmagnani, R. Byrne, C. Slater, K.T. Lau, D. Diamond, Photonic modulation of surface properties: a novel concept in chemical sensing, J. Phys. D-Appl. Phys., 40 (2007) 7238-7244.

[10] S. Scarmagnani, Z. Walsh, C. Slater, N. Alhashimy, B. Paull, M. Macka, D. Diamond, Polystyrene bead-based system for optical sensing using spiropyran photoswitches, J. Mater. Chem., 18 (2008) 5063-5071.

[11] V.I. Minkin, Photo-, thermo-, solvato-, and electrochromic spiroheterocyclic compounds, Chem. Rev., 104 (2004) 2751-2776.

[12] N. Shao, X. Zhang, R. Yang, Applications of Spiropyran Derivatives in Analytical Chemistry, Prog. Chem., 23 (2011) 842-851.

[13] A.V. Chernyshev, A.V. Metelitsa, E.B. Gaeva, N.A. Voloshin, G.S. Borodkin, V.I. Minkin, Photoand thermochromic cation sensitive spiro indoline-pyridobenzopyrans, J. Phys. Org. Chem., 20 (2007) 908-916.

[14] J.Q. Ren, H. Tian, Thermally stable merocyanine form of photochromic spiropyran with aluminum ion as a reversible photo-driven sensor in aqueous solution, Sensors, 7 (2007) 3166-3178.

[15] S. Yagi, S. Nakamura, D. Watanabe, H. Nakazumi, Colorimetric sensing of metal ions by bis(spiropyran) podands: Towards naked-eye detection of alkaline earth metal ions, Dyes Pigm., 80 (2009) 98-105.

[16] S.L. Han, Y. Chen, Mercury ion induced activation of the C-O bond in a photo-responsive spiropyran, Dyes Pigm., 88 (2011) 235-239.

[17] J.F. Zhu, H. Yuan, W.H. Chan, A.W.M. Lee, A colorimetric and fluorescent turn-on chemosensor operative in aqueous media for $\mathrm{Zn}(2+)$ based on a multifunctionalized spirobenzopyran derivative, Org. Biomol. Chem., 8 (2010) 3957-3964. 
[18] N. Shao, J. Jin, H. Wang, J. Zheng, R. Yang, W. Chan, Z. Abliz, Design of Bis-spiropyran Ligands as Dipolar Molecule Receptors and Application to in Vivo Glutathione Fluorescent Probes, J. Am. Chem. Soc., 132 (2010) 725-736.

[19] Y. Liu, M. Fan, S. Zhang, X. Sheng, J. Yao, Basic amino acid induced isomerization of a spiropyran: Towards visual recognition of basic amino acids in water, New J. Chem., 31 (2007) 18781881.

[20] N. Shao, J.Y. Jin, S.M. Cheung, R.H. Yang, W.H. Chan, T. Mo, A spiropyran-based ensemble for visual recognition and quantification of cysteine and homocysteine at physiological levels, Angew. Chem.-Int. Edit., 45 (2006) 4944-4948.

[21] J. Andersson, S.M. Li, P. Lincoln, J. Andreasson, Photoswitched DNA-binding of a photochromic spiropyran, J. Am. Chem. Soc., 130 (2008) 11836-11837.

[22] N. Shao, H. Wang, X.D. Gao, R.H. Yang, W.H. Chan, Spiropyran-Based Fluorescent Anion Probe and Its Application for Urinary Pyrophosphate Detection, Anal. Chem., 82 (2010) 4628-4636.

[23] Y. Shiraishi, M. Itoh, T. Hirai, Colorimetric response of spiropyran derivative for anions in aqueous or organic media, Tetrahedron, 67 (2011) 891-897.

[24] A. Botrel, B. Aboab, F. Corre, F. Tonnard, A theoretical investigation of solvatochromism Application to merocyanines similar to colored forms obtained by flash-photolysis of spiropyrans, Chem. Phys., 194 (1995) 101-116.

[25] F. Benito-Lopez, S. Scarmagnani, Z. Walsh, B. Paull, M. Macka, D. Diamond, Spiropyran modified micro-fluidic chip channels as photonically controlled self-indicating system for metal ion accumulation and release, Sens. Actuators, B 140 (2009) 295-303.

[26] R. Rosario, D. Gust, M. Hayes, F. Jahnke, J. Springer, A.A. Garcia, Photon-modulated wettability changes on spiropyran-coated surfaces, Langmuir, 18 (2002) 8062-8069.

[27] S.-R. Keum, S.-M. Ahn, S.-J. Roh, S.-Y. Ma, The synthesis and spectroscopic properties of novel, photochromic indolinobenzospiropyran-based homopolymers prepared via ring-opening metathesis polymerization, Dyes Pigm., 86 (2010) 74-80.

[28] S. Samanta, J. Locklin, Formation of photochromic spiropyran polymer brushes via surfaceinitiated, ring-opening metathesis polymerization: Reversible photocontrol of wetting behavior and solvent dependent morphology changes, Langmuir, 24 (2008) 9558-9565.

[29] P. Uznanski, UV-assisted formation of nanoaggregates from photochromic spiropyrans in nonpolar solvents, Langmuir, 19 (2003) 1919-1922.

[30] D. Dattilo, L. Armelao, G. Fois, G. Mistura, M. Maggini, Wetting properties of flat and porous silicon surfaces coated with a spiropyran, Langmuir, 23 (2007) 12945-12950.

[31] R. Delgado-Macuil, M. Rojas-Lopez, V.L. Gayou, A. Orduna-Diaz, J. Diaz-Reyes, ATR spectroscopy applied to photochromic polymer analysis, Mater. Charact., 58 (2007) 771-775.

[32] Y. Futami, M.L.S. Chin, S. Kudoh, M. Takayanagi, M. Nakata, Conformations of nitro-substituted spiropyran and merocyanine studied by low-temperature matrix-isolation infrared spectroscopy and density-functional-theory calculation, Chem. Phys. Lett., 370 (2003) 460-468.

[33] G. Cottone, R. Noto, G. La Manna, S.L. Fornili, Ab initio study on the photoisomers of a nitrosubstituted spiropyran, Chem. Phys. Lett., 319 (2000) 51-59.

[34] C.T. Zhao, M.D. Ribeiro, M.F. Portela, Addition polymerisation of 5-vinyl-2-norbornene with nickel bis(acetyl acetonate)/methylaluminoxane system, Journal of Molecular Catalysis a-Chemical, 185 (2002) 81-85.

[35] K.H. Fries, J.D. Driskell, S. Samanta, J. Locklin, Spectroscopic Analysis of Metal Ion Binding in Spiropyran Containing Copolymer Thin Films, Anal. Chem., 82 (2010) 3306-3314.

[36] M.D. Miller, G.L. Baker, M.L. Bruening, Polymer-brush stationary phases for open-tubular capillary electrochromatography, J. Chromatogr. A, 1044 (2004) 323-330.

[37] C. Ventura, R. Byrne, F. Audouin, A. Heise, Atom Transfer Radical Polymerization Synthesis and Photoresponsive Solution Behavior of Spiropyran End-Functionalized Polymers as Simplistic Molecular Probes, J. Polym. Sci., Part A-Polym. Chem., 49 (2011) 3455-3463.

[38] A. Samoladas, D. Bikiaris, T. Zorba, K.M. Paraskevopoulos, A. Jannakoudakis, Photochromic behavior of spiropyran in polystyrene and polycaprolactone thin films - Effect of UV absorber and antioxidant compound, Dyes Pigm., 76 (2008) 386-393.

[39] J.S. Lin, H.T. Chiu, Photochromic behavior of spiropyran and fulgide in thin films of blends of PMMA and SBS, J. Polym. Res.-Taiwan, 10 (2003) 105-110.

[40] F.B. Myers, L.P. Lee, Innovations in optical microfluidic technologies for point-of-care diagnostics, Lab Chip, 8 (2008) 2015-2031. 
[41] S. Stitzel, R. Byrne, D. Diamond, LED switching of spiropyran-doped polymer films, J. Mater. Sci., 41 (2006) 5841-5844.

[42] X.L. Li, J.L. Li, Y.M. Wang, T. Matsuura, J.B. Meng, Synthesis of functionalized spiropyran and spirooxazine derivatives and their photochromic properties, J. Photochem. Photobiol., A 161 (2004) 201-213.

[43] G. Baillet, G. Giusti, R. Guglielmetti, Comparative photodegradation study between spiro indoline and spiro indoline pyran derivatives in solution, J. Photochem. Photobiol., A 70 (1993) 157-161.

[44] G. Baillet, M. Campredon, R. Guglielmetti, G. Giusti, C. Aubert, Dealkylation of N-substituted indolinospironaphthoxazine photochromic compounds under UV irradiation, J. Photochem. Photobiol., A 83 (1994) 147-151.

[45] H. Gorner, Photochromism of nitrospiropyrans: effects of structure, solvent and temperature, Phys. Chem. Chem. Phys., 3 (2001) 416-423.

\section{Biographies}

Larisa Florea studied organic chemistry and chemical engineering at University "Politehnica" from Timisoara, Romania (BSc Hons 2009). In 2009 she joined the Adaptive Sensors Group at Dublin City University where she is currently pursuing a $\mathrm{PhD}$ degree under the supervision of Prof. Dermot Diamond and Dr. Fernando Benito-Lopez. Her research interests include the development of stimuliresponsive polymers as novel sensing materials in micro-fluidics.

Fernando Benito López studied chemistry at the Universidad Autonoma de Madrid and completed his master studies in the Department of Inorganic Chemistry in 2002. He obtained his PhD at the University of Twente, The Netherlands, under the supervision of Prof. David N. Reinhoudt and Dr. Willem Verboom in 2007. He carried out his postdoctoral research in the group of Prof. Dermot Diamond at Dublin City University, Dublin, Ireland. From 2010, he is Team Leader in polymer microfluidics at CLARITY: Centre for Sensor Web Technology, National Centre for Sensor Research, Dublin City University.

Alexandre Hennart studied chemistry at the University of Namur, Belgium. In 2011, he obtained his Master degree in Chemistry and performed a research work on "n-dodecanethiol Self-Assembled Monolayers on Cobalt". The same year during a stay at Adaptive Sensors Group at Dublin City University, he worked under the supervision of Prof. Dermot Diamond and Dr. Fernando Benito-Lopez on the development of reversible spiropyran based sensors. He is presently preparing a PhD under the supervision of Prof. Z. Mekhalif at the Laboratory of Chemistry and Electrochemistry of Surfaces (CES) - University of Namur.

Dermot Diamond received his PhD and DSc from Queen's University Belfast (Chemical Sensors, 1987, Internet Scale Sensing, 2002), and was VP for Research at Dublin City University (2002-2004). He has published over 200 peer-reviewed papers in international journals, is a named inventor in 13 patents, and is co-author and editor of three books. He is currently director of the National Centre for Sensor Research (www.ncsr.ie) and a Principle Investigator in CLARITY (www.clarity-centre.com/), a major research initiative focused on wireless sensor networks. In 2002 he was awarded the inaugural silver medal for Sensor Research by the RSC, London.

*Corresponding author: Tel.: +3531 7007603 fax: +353 17007995 .

E-mail addresses: fernando.lopez@dcu.ie (F. Benito-Lopez), larisa.florea2@mail.dcu.ie (L. Florea), alexandre.hennart@gmail.com (A. Hennart), dermot.diamond@dcu.ie (D. Diamond). 